\title{
Os sentidos da reunião de equipe de uma unidade de internação pediátrica: Compreensão de profissionais e gestoras
}

\section{Aquiles Dacêncio Pereira1, Danielle Abdel Massih Pio', Ana Carolina Nonato ${ }^{1}$, Silvia Franco da Rocha Tonhom ${ }^{1}$, Ione Ferreira Santos ${ }^{1} e$ Maria Elizabeth da Silva Hernandes Correa ${ }^{2}$}

\author{
1 Faculdade de Medicina de Marília, Brasil | psicologoaquiles@gmail.com \\ danimassihpio@hotmail.com; nonato.anacarolina@gmail.com; siltonhom@gmail.com; \\ ionefs13@gmail.com | https://orcid.org/0000-0001-5188-6145; https://orcid.org/0000-0003 \\ 0738-4601; https://orcid.org/0000-0003-2992-068X; https://orcid.org/0000-0001-7522-2861; \\ https://orcid.org/0000-0002-2030-4541 \\ 2 Universidade de Marília, Unimar, Brasil | meshco@gmail.com | https://orcid.org/0000-0003- \\ 2301-714X
}

Resumo: Introdução: A atuação em equipe é considerada um processo de trabalho para executar atividades de modo mais eficiente do que quando realizadas individualmente. Na área da saúde, esta proposta contribui para reorganizar o trabalho, com assistência mais integral e resolutiva aos usuários. Assim, a existência de reuniões de equipe de saúde instituídas formalmente pode garantir maior integralidade da atenção. Objetivos: Compreender quais são as percepções e vivências acerca da reunião de equipe para profissionais e gestoras de uma Unidade de Internação de Pediatria Clínica (UIPC). Métodos: Estudo de vertente qualitativa, organizado em dois momentos: Grupo Focal com os integrantes da reunião de equipe, totalizando nove profissionais, e entrevistas individuais por meio de roteiro semiestruturado com as gestoras, totalizando quatro profissionais. A análise dos dados ocorreu sob a ótica da Análise de Conteúdo - modalidade temática. Resultados: Os profissionais e as gestoras entendem como muito importante a manutenção e a ampliação do espaço da reunião de equipe, considerado referência para o cuidado em saúde ao possibilitar reflexões sobre o trabalho e as necessidades de saúde dos usuários, com maior resolutividade das intervenções. Entretanto, identificam entraves para este processo, uma vez que é necessário estar aberto à escuta e considerar o saber do outro. Também compreendem que a participação dos estudantes é imprescindível para o aprendizado acerca da integralidade do cuidado e que a gestão constitui uma importante aliada para valorização deste espaço. Conclusões: Considerando a pesquisa qualitativa, a coleta dos dados por meio das técnicas de Grupo Focal e de entrevista semiestruturada propiciou reflexões que possibilitaram ao grupo elencar estratégias de enfrentamento a obstáculos e identificar os benefícios do processo de reunião de equipe, com acompanhamento dinâmico da gestão e valorização deste processo de construção.

Palavras-chave: Equipe de Assistência ao Paciente; Gestão de Recursos da Equipe de Assistência à Saúde; Necessidades e Demandas de Serviços de Saúde; Assistência Hospitalar; Pesquisa Qualitativa.

\section{Team Meeting of a Pediatric Inpatient Unit: Meaning and Understanding of Professionals} and Managers

Abstract: Introduction: Team working is a complex of actions to perform a task more efficiently than when performed individually. In health, this process contributes to the reorganization of work, with more resolving and comprehensive assistance to users. Thus, understanding the implications of the form the health team meeting is instituted can guarantee a more comprehensive care to the user. Objectives: to understand what the perceptions and experiences regarding the team meeting for professionals and managers of a Clinical Pediatric Inpatient Unit (UIPC) are. Methodology: Qualitative study, divided into two moments: Focus Group with the members of the team meeting, totaling nine professionals, and individual interviews through a semi-directed script with the managers, totaling four professionals. Data analysis took place from the perspective of Content Analysis - thematic modality. Results: professionals and managers consider important the maintenance and expansion of the team meeting space, considered a reference for health care, as it allows reflections on the work and health needs of users, with greater resolution of the interventions. However, they identify obstacles to this process since it is necessary to be open to listening and considering the knowledge of others. They also understand that the participation of students is important for understanding the integrality of care and that management is an important ally for the valorization of this space. Conclusion: Considering the qualitative research, the collection of data using the Focus Group and semi-directed interview techniques provided reflections that enabled the group to list obstacle-coping strategies and identify the benefits of the team meeting process, with dynamic management monitoring and enhancement of this construction process.

Keywords: Patient Care Team; Crew Resource Management; Healthcare; Health Services Needs and Demand; Hospital Care; Qualitative Research. 


\section{Introdução}

A atuação em equipe pode ser considerada um processo de trabalho para executar atividades de forma mais eficiente do que quando realizadas individualmente (Peduzzi et al., 2020). Na área da saúde, este modelo contribui para o processo de reorganização do trabalho, com assistência mais integral e resolutiva aos usuários (Brasil \& Ministério da Saúde, 2017; Carnut, 2017).

O conceito de equipe multidisciplinar caracteriza-se como um grupo de diferentes profissionais que interagem de forma dinâmica e interdependente e cujo objetivo comum é o cuidado (Horlait et al., 2019), conceito que vai além de um conjunto de diferentes profissionais que trabalham isoladamente.

A organização das equipes está associada à complexidade da demanda de cuidado aos usuários (Araújo \& Assis, 2017). Nessas situações, muitos profissionais se deparam com seus próprios limites, encontrando subsídios para compreensão e atendimento dos casos mais vulneráveis nos colegas com outras formações. Além disso, a prática do trabalho em equipe traz seus próprios desafios: requer competências e habilidades interpessoais, além de justificação clara e objetiva das ações de cuidado propostas (Valentim et al., 2020).

Em relação aos novos profissionais, considerando o aumento da compreensão dos determinantes do processo saúde-doença e a criação do Sistema Único de Saúde (SUS) por meio da Constituição Federal de 1988, o ensino em saúde sofre modificações para se alinhar à Lei de Diretrizes e Bases da Educação Nacional, instituída em 1996, e às Diretrizes Curriculares Nacionais de cada curso da saúde (Costa et al., 2018).

Considerando a educação um ato coletivo e comprometido com a interação e a troca entre pessoas, os novos modelos de ensino visam romper lógicas dualistas, possibilitando articular teoria e prática (Mitre et al., 2008) e desenvolver habilidades colaborativas que fortaleçam o trabalho em equipe para a transformação das ações de saúde (Peduzzi et al., 2013). Deste modo, estes novos modelos de ensino produzem modificações significativas na forma e no conteúdo das reuniões de equipe em ambientes hospitalares, contemplando o modelo de cuidado integral (Costa et al., 2018).

Nesse sentido, tem-se a inquietação de como ocorrem as reuniões de equipe e se esse processo contribui para a formação de profissionais e para o cuidado em saúde dos usuários, promovendo construção coletiva da atenção em uma Unidade de Internação Hospitalar. Assim, o presente estudo objetivou compreender a percepção e as vivências sobre a reunião de equipe na perspectiva de profissionais e gestoras de uma Unidade de Internação de Pediatria Clínica (UIPC).

\section{Metodologia}

Este estudo tem delineamento qualitativo, que valoriza significados, representações e intencionalidades das relações humanas, traduzindo estes aspectos para a ciência (Minayo et al., 2016). O ser humano é uma entidade social, que apreende o lugar que ocupa e as ações que realiza, compreendendo o sentido de suas vivências e expressandoas pela linguagem, um movimento repleto de interpretações e reflexões embasadas em suas caraterísticas socioculturais (Minayo, 2012; Minayo et al., 2016).

A pesquisa foi realizada em uma Unidade de Internação de Pediatria Clínica (UIPC) de um hospital-escola vinculado a uma faculdade de medicina e enfermagem do interior do estado de São Paulo, integrado ao SUS por meio da Secretaria Estadual da Saúde e do Departamento Regional de Saúde (DRS) IX (nove). Esta unidade é constituída de 14 leitos e possui 62 municípios em sua área de abrangência, totalizando aproximadamente 1.200.000 habitantes atendidos (Hospital das Clínicas de Marília, 2018a, 2018b). 
Nesta UIPC, a reunião de equipe é composta apenas pelos profissionais diretamente relacionados aos cuidados dos usuários, não contando com a participação das gestoras. Para abranger a percepção dos dois grupos, a escolha dos participantes se deu de forma intencional: o primeiro grupo foi composto por profissionais que participavam das reuniões de equipe, como médicos, enfermeiros, técnicos de enfermagem, psicólogos, fonoaudiólogos, fisioterapeutas, terapeutas ocupacionais, nutricionistas, pedagogos, farmacêuticos, assistentes sociais e residentes médicos, cujos dados foram coletados por meio da técnica do Grupo Focal; o segundo, pelas gestoras desta unidade assistencial, cujos dados foram coletados por meio de entrevistas individuais, orientadas por roteiro semiestruturado.

Como critério de inclusão para o primeiro grupo, foram considerados os profissionais que participam da reunião de equipe daquela UIPC e que estivessem na prática profissional há pelo menos um ano, totalizando nove trabalhadores; para o segundo grupo, consideraram-se todas as gestoras daquela unidade assistencial, totalizando quatro participantes. Foram excluídos os profissionais que não puderam estar presentes no momento da coleta de dados e também estudantes de medicina e enfermagem e residentes multiprofissionais, que não compunham o campo de estágio no momento da coleta de dados. Deste modo, considerando o cenário em que a pesquisa se insere, o total de nove profissionais da UIPC e quatro gestoras da unidade hospitalar em que está contida foi suficiente para que as percepções deste coletivo fossem analisadas e apreendidas.

O Grupo Focal é uma técnica derivada da psicologia social e seu uso está pautado na seleção e reunião de atores que possuam características em comum para discussão de suas vivências (Turato, 2013). O termo "Focal" se deve ao fato de envolver algum tipo de atividade intencional e coletiva que, no caso deste estudo, é a discussão acerca da reunião de equipe. Ele possui uma vantagem em relação às demais técnicas de coleta de dados, pois o Grupo Focal evidencia a dinâmica de funcionamento das pessoas na realidade, espelhando, na esfera microssocial, o modo de pensar e interagir que acontece na esfera macrossocial. Outra vantagem é seu uso em estudos em que os participantes possuem diferentes formações e posições hierárquicas nas instituições, buscando variedade de percepções (Backes et al., 2011; Nóbrega et al., 2016; Souza, 2020; Turato, 2013).

O facilitador da atividade precisa ser cuidadoso em sua participação para não interferir indevidamente no processo do grupo, com opiniões, conclusões, sínteses ou proposições de ideias, mas também deve cuidar para que ocorram reais trocas entre os participantes, que os objetivos do trabalho sejam alcançados e as ideias levantadas e discutidas tenham o devido encaminhamento. Neste sentido, a função de "facilitar" deve garantir que a discussão ocorra, tornando o grupo protagonista de suas interações, reflexões e conclusões. O maior cuidado a se tomar nesta técnica é não a transformar em uma entrevista em grupo, baseada apenas na troca de perguntas e respostas entre o facilitador e os participantes, e não no processo grupal como criador de sua própria dinâmica (Souza, 2020; Turato, 2013).

O Grupo Focal foi realizado em um encontro em outubro de 2019, com duração de aproximadamente uma hora, e foi orientado por um roteiro semiestruturado elaborado pelos autores e que contemplava um conjunto de perguntas fechadas e quatro conjuntos de perguntas abertas. O primeiro conjunto contempla os dados de identificação, como nome, idade, gênero, categoria profissional, tempo de atuação em pediatria e se era docente, assistente, pós-graduando ou graduando, com o objetivo de caracterização dos participantes; os demais conjuntos contemplam os núcleos "Compreensão do que é equipe", "Compreensão do que é reunião", "Utilidade e sentido da reunião" e "Implementações", com perguntas abertas que guiaram o processo grupal. Os dados foram audiogravados e transcritos para posterior análise. Além disso, a pesquisadora que orientou o andamento do grupo realizou anotações em diário de campo. O segundo momento deste estudo foi a participação das gestoras da unidade assistencial em questão por meio de entrevistas individuais semiestruturadas, realizadas entre maio de 2019 a maio de 2020, a depender da disponibilidade de cada participante, que foram audiogravadas e transcritas na íntegra para posterior análise. 
O roteiro de entrevista continha os dados de identificação dos participantes, como nome, idade, gênero, categoria profissional e qual seu cargo na gestão, e perguntas abertas, de modo a problematizar a percepção destes sobre o processo, a operacionalização e a contribuição da reunião de equipe para a prática e para a formação dos profissionais, considerando sua importância, suas potências, suas fragilidades e sua influência nos indicadores hospitalares. Além disso, as gestoras também foram indagadas se gostariam de propor melhorias para este espaço.

A análise dos dados obtidos nestes dois momentos ocorreu sob a óptica da Análise de Conteúdo - modalidade temática, que consiste em um conjunto de técnicas para a análise dos dados brutos por meio de procedimentos sistemáticos para a descrição dos conteúdos das mensagens, permitindo inferência de conhecimentos em relação às suas condições de produção e recepção por dedução lógica. Deste modo, objetiva-se evitar incertezas, com enriquecimento da leitura daqueles dados. Esta metodologia é constituída de três etapas: pré-análise, exploração do material e tratamento dos resultados (Bardin, 2016; Minayo et al., 2016).

A pré-análise transforma o material bruto em operacional por meio de sucessivas leituras para um aprofundamento cada vez maior, de modo que o material seja compreendido em relação ao conjunto e às suas peculiaridades, e para elencar alguns pressupostos que nortearão a interpretação dos dados e, consequentemente, facilitarão o processo de classificação destes. A metodologia é constituída de outras quatro etapas: leitura flutuante, que estabelece o contato com os documentos de coleta de dados; escolha dos documentos, com definição do que será o objeto de análise; formulação de objetivos e das hipóteses; e elaboração de índices e indicadores, que são os pressupostos norteadores da interpretação. A etapa seguinte consiste na exploração do material, fase de descrição analítica para codificação e identificação das unidades de contexto, momento em que os trechos são efetivamente classificados por meio do agrupamento de dados e apontamento das categorias temáticas. Por fim, ocorre o tratamento dos resultados, etapa que permite a extração de núcleos de sentido das categorias temáticas. Estes são trabalhados de modo a dialogar com os pressupostos norteadores e, se necessário, novos podem ser elencados. Este momento constitui-se de uma fase de análise crítica e reflexiva dos resultados e de seu diálogo com a literatura (Bardin, 2016; Minayo et al., 2016).

Esta pesquisa foi aprovada pelo Comitê de Ética em Pesquisa (CEP) da instituição em 2019, sob parecer 3.250.706.

\section{Resultados}

\subsection{Grupo Focal com Profissionais}

Participaram do Grupo Focal nove profissionais da saúde, todos do gênero feminino: uma enfermeira, duas fisioterapeutas, uma fonoaudióloga, duas médicas, uma pedagoga e duas residentes médicas de pediatria. A faixa etária predominante encontrava-se entre 28 e 37 anos de idade, com tempo de atuação na área pediátrica entre seis e dez anos.

Conforme procedimento metodológico da Análise de Conteúdo, modalidade temática, após a coleta e análise dos dados obtidos, identificaram-se cinco categorias temáticas, conforme a seguir: 
Tabela. 1. Categorias temáticas e núcleos de sentido - Profissionais.

\begin{tabular}{|c|c|}
\hline Categorias temáticas & Núcleos de sentido \\
\hline $\begin{array}{l}\text { Constituição da reunião de } \\
\text { equipe }\end{array}$ & $\begin{array}{l}\text { - Determinação da gestão; } \\
\text { - Dificuldades de compreensão da proposta; } \\
\text { - Processos de Educação Continuada; } \\
\text { - Encontro de pessoas, com um referencial biológico de atenção. }\end{array}$ \\
\hline $\begin{array}{l}\text { Construção do cuidado a } \\
\text { partir da valorização do } \\
\text { trabalho em equipe }\end{array}$ & $\begin{array}{l}\text { - Amadurecimento e valorização da contribuição de todos os profissionais; } \\
\text { - Momento de colegiado entre os profissionais; } \\
\text { - Descoberta das interfaces sociais e psicológicas no cuidado, construindo } \\
\text { uma compreensão mais holística; } \\
\text { - Frequência e horário da reunião baseados na combinação entre a rotina do } \\
\text { serviço com a agenda dos profissionais; } \\
\text { - Práticas colaborativas entre os atores envolvidos nos cuidados às } \\
\text { necessidades ampliadas dos usuários do serviço; } \\
\text { - Participação da família para identificar dificuldades, necessidades e } \\
\text { aspectos socioculturais a fim de melhorar o manejo e a resolutividade dos } \\
\text { casos. }\end{array}$ \\
\hline $\begin{array}{l}\text { Desafios no processo de } \\
\text { atenção }\end{array}$ & $\begin{array}{l}\text { - Aumento na complexidade dos casos e do uso de novas tecnologias; } \\
\text { - Perfil dos usuários do sistema de saúde: posturas e compreensões; } \\
\text { - Transição do cuidado; } \\
\text { - Percepção de ausência e/ou escassez de recursos humanos suficientes } \\
\text { para haver representatividade das categorias profissionais. }\end{array}$ \\
\hline $\begin{array}{l}\text { Desafios no processo de } \\
\text { formação }\end{array}$ & $\begin{array}{l}\text { - Pouca participação, interesse e/ou maturidade dos estudantes quanto ao } \\
\text { espaço da reunião de equipe; } \\
\text { - Horário praticado inviabiliza a agenda de atividades do estudante da } \\
\text { graduação e da residência multiprofissional; } \\
\text { - Necessidade de implementar ações para efetivar a participação do } \\
\text { estudante de Medicina;. }\end{array}$ \\
\hline
\end{tabular}

Considerando a categoria "Constituição da reunião de equipe", os profissionais relatam que esta foi implementada por meio de uma normativa institucional. Na prática, o processo foi organizado por encontros de Educação Continuada, gerando dificuldade de compreensão de seu propósito. Deste modo, a reunião de equipe na UIPC configurou-se, inicialmente, como um "encontro de pessoas" com enfoque biomédico do cuidado; todavia, a regularidade das reuniões promoveu maior convivência entre os participantes, suscitando reflexões e novas necessidades daquele grupo, ampliando o foco do cuidado.

Em relação à categoria "Construção do cuidado a partir da valorização do trabalho em equipe", verificou-se que a reunião de equipe possibilitou que seus integrantes valorizassem a contribuição de todos os profissionais, com melhora da escuta e compartilhamento dos saberes sem imposição.

Considerando categoria "Desafios no processo de atenção", os participantes identificam um aumento na complexidade dos casos e também sinalizam dificuldades em lidar com o perfil dos usuários, pois os familiares não compreendem 0 conceito de corresponsabilização e autonomia do cuidado.

Em relação à categoria "Desafios no processo de formação", alguns profissionais avaliaram pouca participação dos estudantes nas reuniões de equipe, percebendo que isto se deve à falta de interesse e de maturidade dos estudantes, além da dificuldade de organização das demais atividades educacionais para permitir que participem deste espaço. 


\subsection{Entrevistas com Gestoras}

Foram realizadas quatro entrevistas individuais com as gestoras, sendo todas do gênero feminino: três enfermeiras e uma médica. A idade situava-se entre 35 e 40 anos. Duas profissionais eram Diretoras Técnicas; as outras duas, Assessoras Técnicas.

A tabela 2 reúne as categorias temáticas derivadas das entrevistas com gestoras.

Tabela. 2. Categorias temáticas e núcleos de sentido - Gestoras.

\begin{tabular}{ll}
\hline \multicolumn{1}{c}{$\begin{array}{c}\text { Categorias } \\
\text { temáticas }\end{array}$} & \multicolumn{1}{c}{ Núcleos de sentido } \\
\hline $\begin{array}{l}\text { Um espaço de } \\
\text { referência para o } \\
\text { cuidado em } \\
\text { saúde }\end{array}$ & $\begin{array}{l}\text { - Potencializa a qualificação da equipe e do cuidado em saúde; } \\
\text { - Favorece a elaboração de um plano terapêutico. }\end{array}$ \\
$\begin{array}{ll}\text { O que dificulta a } \\
\text { constituição } \\
\text { desse espaço }\end{array}$ & $\begin{array}{l}\text { - Comunicação disfuncional; } \\
\text { - Fragilidade na formação dos profissionais quanto ao processo de trabalho em } \\
\text { - Manutenção do modelo predominantemente biomédico; }\end{array}$ \\
\hline $\begin{array}{l}\text { - Pouca valorização e apropriação dos profissionais e estudantes. } \\
\text { O que fortalece a } \\
\text { constituição } \\
\text { desse espaço }\end{array}$ & $\begin{array}{l}\text { - Promove melhor organização dos processos de trabalho; } \\
\text { - Favorece a mudança de um modelo de atenção hegemônico; }\end{array}$ \\
\hline
\end{tabular}

Considerando a categoria "Um espaço de referência para o cuidado em saúde", as gestoras identificam que as reuniões de equipe incrementam a qualificação dos profissionais e a qualidade do cuidado em saúde, com integração multidisciplinar.

Em relação à categoria "O que dificulta a constituição desse espaço", apontaram a comunicação disfuncional, que gera conflitos entre os profissionais, e o modelo de atenção biomédico, pois mesmo quando o profissional de medicina não está presente, sua posição direciona a decisão final, prejudicando o cuidado em saúde.

Considerando a categoria "O que fortalece a constituição desse espaço", as gestoras apontaram que as reuniões fortalecem a relação entre profissionais, constituindo-se como um momento de discussões conjuntas. Outro fortalecimento identificado foi a articulação com a gestão, gerando impacto no cuidado e nos indicadores hospitalares.

\section{Discussão}

Nesta UIPC, o movimento para estabelecimento da reunião de equipe iniciou-se com uma lógica distante da construção compartilhada dos sujeitos, o que foi identificado como um obstáculo inicial à consolidação deste momento; todavia, com maior regularidade e envolvimento dos sujeitos, suscitou maior engajamento e valorização da contribuição de seus integrantes, reafirmando que a reunião de equipe é um importante momento para organização do trabalho, com intensas reflexões dos profissionais sobre suas vivências (Fox \& Reeves, 2015; Peduzzi \& Agreli, 2018), devendo possibilitar o planejamento em conjunto das ações (Santos et al., 2017b), com organização horizontal do trabalho em relação às condutas e às tarefas da equipe (Peduzzi \& Agreli, 2018).

A presença de profissionais em formação foi avaliada como necessária, porém praticamente inexistente por diversos fatores dos próprios graduandos e da organização curricular. Além disso, participantes identificaram dificuldades para lidar com a mudança na complexidade dos casos e no perfil dos usuários e de seus familiares. 
Para atenção à saúde com qualidade, é importante a integração ensino-serviço, proporcionando uma formação acadêmica coesa com a prática profissional e com as necessidades de saúde da população (Zarpelon et al., 2018), e que os profissionais atuem de modo colaborativo para maior resolutividade dos casos (Santos et al., 2017a).

As gestoras consideram as reuniões de equipe como espaço de qualificação profissional e do cuidado em saúde, corroborando com a definição de que o espaço da reunião possibilita o encontro dos profissionais para compartilhamento de informações, planejamento de intervenções em saúde e debate de questões administrativas (Santos et al., 2017a). Todavia, consideram algumas dificuldades para estabelecimento deste espaço, como a comunicação disfuncional e o modelo biomédico. Nesse sentido, verificase que a comunicação é norteadora das intervenções, mas pode ser instrumento construtivo ou de ações mecânicas (Santos et al., 2017b) e que a efetiva relação interprofissional requer que todos os profissionais se envolvam no cuidado e contribuam com os espaços coletivos de construção (Peduzzi \& Agreli, 2018).

Como fortalezas deste espaço, as gestoras apontaram melhoria nas relações interprofissionais e na articulação com a gestão, com impactos no cuidado e nos indicadores hospitalares. Essa prática é considerada potencializadora da mudança do modelo de atenção pela contribuição dos diversos profissionais, viabilizando ações de cuidado mais resolutivas e humanizadas (Cardoso \& Hennington, 2011); assim, se houver valorização por parte dos gestores, as equipes tendem a conduzir o trabalho de forma mais engajada, tornando as reuniões de equipe um momento de crescimento, com melhoria dos processos de trabalho (Organização Mundial de Saúde, 2010).

\section{Conclusões}

Considerando a pesquisa qualitativa, a coleta dos dados por meio do Grupo Focal oportunizou ao grupo reflexões que os possibilitaram elencar estratégias de enfrentamento a obstáculos e benefícios do processo de reunião de equipe. Com relação ao grupo de gestoras, a técnica de entrevista semiestruturada propiciou reflexões sobre sua importância no movimento de reunião de equipe por meio de um acompanhamento dinâmico, estratégico e acolhedor, com valorização deste processo de construção.

Os profissionais e as gestoras entendem como muito importante a manutenção e a ampliação do espaço da reunião de equipe, considerado referência para o processo de trabalho em grupo, pois poderão ressignificar suas ações para que a formação interprofissional seja inerente à prática verdadeiramente colaborativa, com maior resolutividade das intervenções, e também compreendem que a participação dos estudantes é importante para o entendimento da integralidade do cuidado e o fortalecimento da formação. Entretanto, identificam entraves em todo o processo, como o modelo biomédico e a comunicação disfuncional. Para potencializar este espaço, é necessário fortalecer a integração entre todos os sujeitos envolvidos, gestão, assistência, população e academia, com foco na integralidade do cuidado ao usuário.

\section{Referências}

Araújo, P. O., \& Assis, M. M. A. (2017). Organização da demanda e oferta de serviços na Estratégia Saúde da Família. Saúde.Com, 13(4). https://doi.org/10.22481/rsc.v13i4.470

Backes, D. S., Colomé, J. S., Erdmann, R. H., \& Lunardi, V. L. (2011). Grupo focal como técnica de coleta e análise de dados em pesquisas qualitativas. O Mundo Da Saúde, 35(4), 438-442. https://doi.org/10.15343/0104-7809.2011354438442

Bardin, L. (2016). Análise de Conteúdo. Edições 70.

Brasil, \& Ministério da Saúde. (2017). Portaria n. 2436, de 21 de setembro de 2017. Aprova a Política Nacional de Atenção Básica, estabelecendo a revisão de diretrizes para a organização da Atenção Básica, no âmbito do Sistema Único de Saúde (SUS).

https://bvsms.saude.gov.br/bvs/saudelegis/gm/2017/prt2436_22_09_2017.html 
Cardoso, C. G., \& Hennington, É. A. (2011). Trabalho em equipe e reuniões multiprofissionais de saúde: uma construção à espera pelos sujeitos da mudança. Trabalho, Educação e Saúde, 9(suppl 1), 85-112. https://doi.org/10.1590/S1981-77462011000400005

Carnut, L. (2017). Cuidado, integralidade e atenção primária: articulação essencial para refletir sobre o setor saúde no Brasil. Saúde Em Debate, 41(115), 1177-1186. https://doi.org/10.1590/01031104201711515

Costa, D. A. S., Silva, R. F., Lima, V. V., \& Ribeiro, E. C. O. (2018). Diretrizes curriculares nacionais das profissões da Saúde 2001-2004: análise à luz das teorias de desenvolvimento curricular. Interface - Comunicação, Saúde, Educação, 22(67), 1183-1195. https://doi.org/10.1590/180757622017.0376

Fox, A., \& Reeves, S. (2015). Interprofessional collaborative patient-centred care: a critical exploration of two related discourses. Journal of Interprofessional Care, 29(2), 113-118. https://doi.org/10.3109/13561820.2014.954284

Horlait, M., Baes, S., Dhaene, S., Van Belle, S., \& Leys, M. (2019). How multidisciplinary are multidisciplinary team meetings in cancer care? An observational study in oncology departments in Flanders, Belgium. Journal of Multidisciplinary Healthcare, Volume 12, 159-167. https://doi.org/10.2147/JMDH.S196660

Hospital das Clínicas de Marília. (2018a). Materno Infantil. HC Famema. http://testehc.famema.br/materno-infantil/

Hospital das Clínicas de Marília. (2018b). Unidades Assistenciais. HC Famema. http://hc.famema.br/unidades-assistenciais/

Minayo, M. C. de S. (2012). Análise qualitativa: teoria, passos e fidedignidade. Ciência \& Saúde Coletiva, 17(3), 621-626. https://doi.org/10.1590/S1413-81232012000300007

Minayo, M. C. de S., Deslandes, S. F., \& Gomes, R. (2016). Pesquisa Social: teoria, método e criatividade. Editora Vozes.

Mitre, S. M., Siqueira-Batista, R., Girardi-de-Mendonça, J. M., Morais-Pinto, N. M. de, Meirelles, C. de A. B., Pinto-Porto, C., Moreira, T., \& Hoffmann, L. M. A. (2008). Metodologias ativas de ensinoaprendizagem na formação profissional em saúde: debates atuais. Ciência \& Saúde Coletiva, 13(suppl 2), 2133-2144. https://doi.org/10.1590/S1413-81232008000900018

Nóbrega, D. O., Andrade, E. dos R. G., \& Melo, E. S. do N. (2016). Pesquisa com Grupo Focal: contribuições ao estudo das Representações Sociais. Psicologia \& Sociedade, 28(3), 433-441. https://doi.org/10.1590/1807-03102016v28n3p433

Organização Mundial de Saúde. (2010). Marco para Ação em Educação Interprofissional e Prática Colaborativa. Editora Freelance.

Peduzzi, M., \& Agreli, H. F. (2018). Trabalho em equipe e prática colaborativa na Atenção Primária à Saúde. Interface - Comunicação, Saúde, Educação, 22(suppl 2), 1525-1534. https://doi.org/10.1590/1807-57622017.0827

Peduzzi, M., Agreli, H. L. F., Silva, J. A. M. da, \& Souza, H. S. de. (2020). Trabalho em equipe: uma revisita ao conceito e a seus desdobramentos no trabalho interprofissional. Trabalho, Educação e Saúde, 18(suppl 1). https://doi.org/10.1590/1981-7746-sol00246

Peduzzi, M., Norman, I. J., Germani, A. C. C. G., Silva, J. A. M. da, \& Souza, G. C. de. (2013). Educação interprofissional: formação de profissionais de saúde para o trabalho em equipe com foco nos usuários. Revista Da Escola de Enfermagem Da USP, 47(4), 977-983. https://doi.org/10.1590/S0080-623420130000400029

Santos, E. O. dos, Coimbra, V. C. C., Kantorski, L. P., Pinho, L. B. de, Andrade, A. P. M. de, \& Eslabão, A. D. (2017a). Reunião de equipe: proposta de organização do processo de trabalho Team meeting: proposal for the work process organization. Revista de Pesquisa: Cuidado é Fundamental Online, 9(3), 606. https://doi.org/10.9789/2175-5361.2017.v9i3.606-613

Santos, E. O., Coimbra, V. C. C., Kantorski, L. P., Pinho, L. B. de, Andrade, A. P. M. de, \& Eslabão, A. D. (2017b). Avaliação da participação dos profissionais na reunião de equipe do centro de atenção psicossocial. Revista de Enfermagem UFPE on Line, 11(12), 5186. https://doi.org/10.5205/19818963-v11i12a22611p5186-5196-2017

Souza, L. K. de. (2020). Recomendações para a Realização de Grupos Focais na Pesquisa Qualitativa. PSI UNISC, 4(1), 52-66. https://doi.org/10.17058/psiunisc.v4i1.13500

Turato, E. R. (2013). Tratado da metodologia da pesquisa clínico-qualitativa: Construção teóricoepistemológica, discussão comparada e aplicação nas áreas da saúde e humanas (6ª). Editora Vozes.

Valentim, L. V., Luz, R. A. de, Costa Santos, L. S., \& Noca, C. R. da S. (2020). Percepção dos profissionais de enfermagem quanto ao trabalho em equipe. Revista Baiana de Enfermagem, 34 https://doi.org/10.18471/rbe.v34.37510

Zarpelon, L. F. B., Terencio, M. L., \& Batista, N. A. (2018). Integração ensino-serviço no contexto das escolas médicas brasileiras: revisão integrativa. Ciência \& Saúde Coletiva, 23(12), 4241-4248. https://doi.org/10.1590/1413-812320182312.32132016 